\title{
Two closely spaced mutations in cis result in Ullrich congenital muscular dystrophy
}

\author{
Hideki Shimomura', Tomoko Lee', Yasuhiko Tanaka', Hiroyuki Awano², Kyoko Itoh', Ichizo Nishino $\mathbb{1}^{4}$ and \\ Yasuhiro Takeshima'
}

\begin{abstract}
A 2-year-old boy was diagnosed with Ullrich congenital muscular dystrophy (UCMD) by muscle biopsy. COL6A3 gene analysis by next-generation sequencing revealed two heterozygous splice-site mutations (c.6283-1 G > G/T and c.6310$2 \mathrm{~A}>\mathrm{A} / \mathrm{T}$ ), whereas normal mRNA was produced. Genomic DNA analysis revealed two mutations located on the same allele; however, no mutation was detected in either parent. These results indicated that two closely spaced de novo mutations resulted in the autosomal dominant UCMD.
\end{abstract}

Ullrich congenital muscular dystrophy (UCMD), a collagen type VI-related disorder resulting from protein defects in the extracellular matrix, is characterized by muscle weakness, hypotonia, proximal joint contractures, and marked hyperlaxity of the distal joints (MIM No. 254090). Another example of a collagen type VI-related disorder is Bethlem myopathy (BM; MIM No. 158810). These conditions arise from mutations in COL6A1 and COL6A2 situated head-to-tail on 21q22.3, as well as in COL6A3 located on 2q37.3. UCMD and BM were previously considered to be an autosomal recessive condition and an autosomal dominant condition, respectively. However, cases of autosomal dominant $\mathrm{UCMD}^{1}$ and autosomal recessive $\mathrm{BM}^{2}$ have also been reported. Currently, both UCMD and BM are known to occur either via autosomal recessive or autosomal dominant mechanisms. Although homozygous and compound heterozygous mutations have been described in patients with both UCMD and BM, no cases with closely spaced de novo mutations in cis have been reported. Here, we report a patient with UCMD carrying two closely spaced de novo mutations in the same COL6A3 allele.

\footnotetext{
Correspondence: Hideki Shimomura (shimomura-hyg@umin.org)

${ }^{1}$ Department of Pediatrics, Hyogo College of Medicine, Nishinomiya, Japan

${ }^{2}$ Department of Pediatrics, Kobe University Graduate School of Medicine, Kobe, Japan

Full list of author information is available at the end of the article.
}

A 2-year-old boy was born normally after 35 weeks of gestation. He exhibited mild developmental delay, with head control at 6 months and walking at 15 months of age. His serum creatine kinase level was $189 \mathrm{U} / \mathrm{L}$. He presented marked distal joint looseness, diminished deep tendon reflexes, and a waddling gait. At 6 years of age, he could not walk by himself. Histochemical analysis and collagen VI immunostaining verified UCMD diagnosis. Nextgeneration sequencing of the genomic DNA revealed two heterozygous splice-site mutations in COL6A3 (viz., c.6283$1 \mathrm{G}>\mathrm{G} / \mathrm{T}$ and c.6310-2 $\mathrm{A}>\mathrm{A} / \mathrm{T}$ ), and mRNA analysis revealed normal as well as aberrantly spliced transcripts. These findings were previously reported in patient 7 in a study, which suggested a dominantly inherited condition ${ }^{3}$.

For COL6A3 mRNA analysis, total RNA was isolated from thin-sliced muscle sections obtained from frozen biopsied muscle tissue. Reverse-transcription polymerase chain reaction (RT-PCR) was employed using a forward primer that corresponded to a segment of exon 15 (c15F: 5'-GA CAACATTGCCGAGAAAGC- $3^{\prime}$ ) and a reverse primer that corresponded to a segment of exon 21 (c21R: 5'-TC GAATCCCAACATCTCCTC-3'). The PCR products were separated by electrophoresis on a $3 \%$ agarose gel. To detect and semiquantify the minor RT-PCR products, the amplified products were analyzed by capillary electrophoresis (3500xL Genetic Analyzer; Applied Biosystems) after a 25cycle PCR amplification using an unlabeled c15F primer and 
FAM-labeled c21R primer. The amount of each product was quantified by measuring the peak areas. The RT-PCR products were then subjected to sequencing after subcloning into a pT7Blue $\mathrm{T}$-vector as described previously ${ }^{4}$.

To determine whether the two mutations were located on the same allele, the nucleotide sequence of the PCR products amplified from genomic DNA using a forward primer on intron 17 (g17F: 5'-AACATTCAAATGGGGT GGAG-3') and reverse primer on intron 19 (g19R: 5'-GA ACCAAAAGCAGTTTGGACTT-3') was determined after subcloning into the pT7Blue $\mathrm{T}$-vector.

To characterize the COL6A3 nucleotide change in the patient's parents, a region encompassing exons 18 and 19 was PCR-amplified from genomic DNA using the g17F and g19R primers. The PCR products were directly sequenced with the BigDye Terminator Cycle Sequencing kit (Amersham Biosciences, Piscataway, NJ, USA) using the automatic DNA sequencer 3500xL Genetic Analyzer.
To analyze the impact of the mutations on splicing probability, we used the in silico prediction tool MaxEntScan with the Weight Matrix Model (http://genes.mit. edu/burgelab/maxent/Xmaxentscan_scoreseq_acc.html). A high score indicated a high possibility of the sequence being a splicing site ${ }^{5}$. The Shapiro splicing probability matrix scores (Shapiro's score) were calculated as described previously ${ }^{6}$. Additionally, in silico analysis for the number of exonic splicing enhancers (ESEs) and intronic splicing enhancers (ISEs) was also performed using the ACESCAN2 Web Server (http://genes.mit.edu/acescan2/) ${ }^{7}$.

Based on previous results, we evaluated COL6A3 mRNA semiquantitatively, including the minor products, and muscle COL6A3 mRNA was analyzed using capillary electrophoresis. Analysis of the muscle-expressing COL6A3 mRNA revealed three kinds of products: a normal mRNA and two larger mRNAs (Fig. 1a, b). Nucleotide sequencing after subcloning revealed that exon 18 was skipped and that

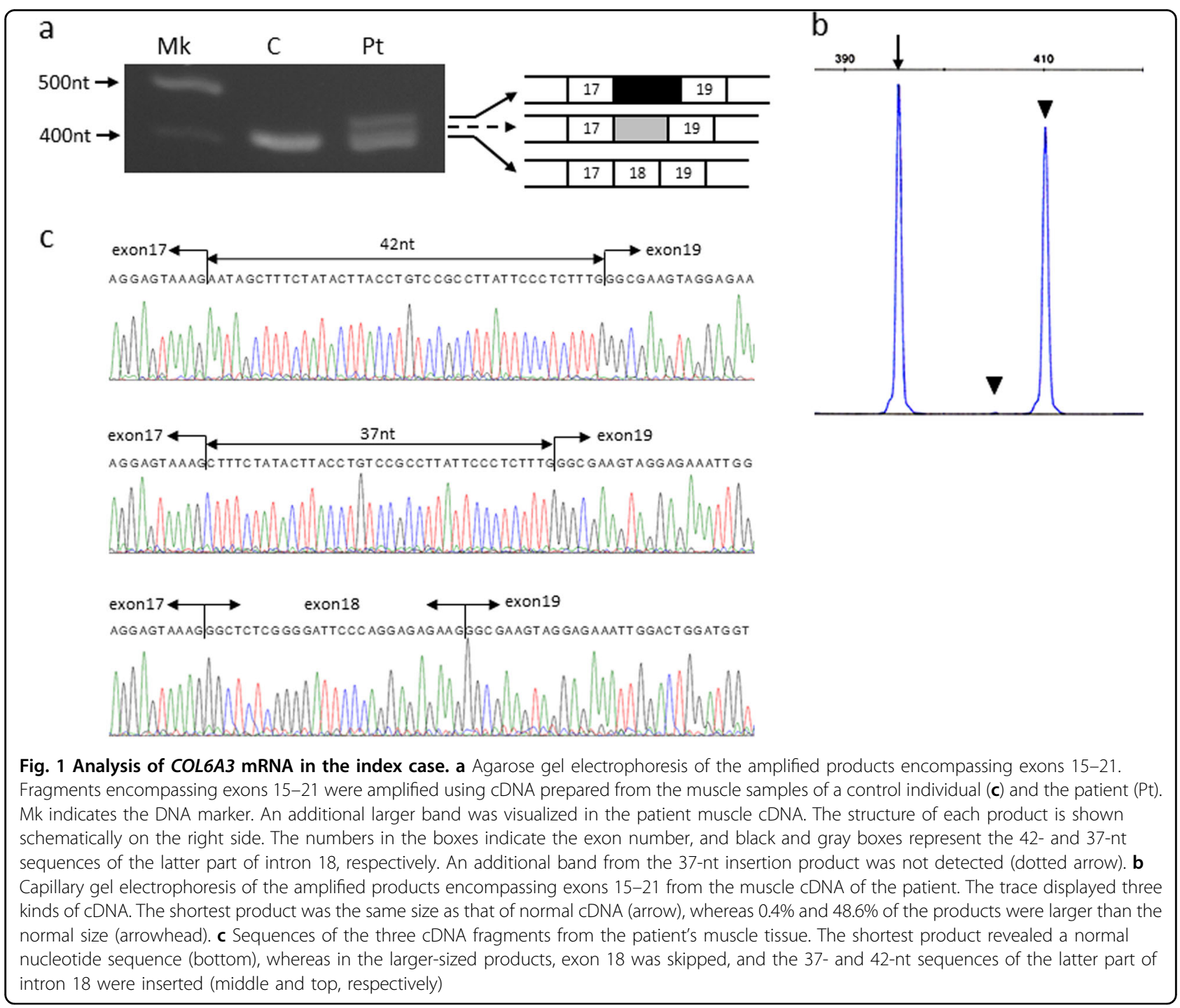


either a 37-nt or a 42-nt sequence of the latter part of intron 18 was inserted into the two larger mRNAs (Fig. 1c). Semiquantitative analysis by capillary electrophoresis revealed that $0.4 \%$ and $48.6 \%$ of mRNAs were included in the 37- and 42-nt sequences, respectively (Fig. 1b).

The genomic DNA sequence was analyzed after subcloning. The results revealed that one allele had a normal sequence, whereas the two closely spaced mutations on the other allele were separated by $137 \mathrm{nt}$. These mutations were not identified in the boy's parents. The findings concluded that there were two closely spaced de novo mutations in the same allele of COL6A3, where c.6283$1 \mathrm{G}>\mathrm{T}$ resulted in the skipping of exon 18 , and c.6310$2 \mathrm{~A}>\mathrm{T}$ activated the cryptic splice sites in the 37 - and 42nt sequences upstream of exon 19 (Fig. 2).

The MaxEntScan score of the splice acceptor site of exon 18 was 9.86 and that of exon 19 was 9.59. As a result of mutation, these scores changed to 1.26 in exon 18 and 1.22 in exon 19. The scores of the cryptic splice sites 37 and 42 upstream of exon 19 were 7.72 and 1.02, respectively (Fig. 2). The Shapiro's score of these splice sites was evaluated as shown in Fig. 2. In silico analysis of ESEs and ISEs revealed that the number of ESEs in exon 18 was 4 and that in exon 19 was 10, while the number of ISEs in intron 18 was 12 and that in intron 19 was 6.

In this report, a case of autosomal dominant UCMD with two closely spaced de novo mutations in the same allele was identified for the first time. Closely spaced multiple mutations (CSMMs) were classified into two groups: those separated by $<100 \mathrm{nt}$ and those separated by $>100 \mathrm{nt}^{8}$. Lampe et al. $(2005,2008)$ reported a large number of mutations related to collagen type VI-related disorders, all of which were single nucleotide substitutions, but none were CSMMs. Chen et al. reviewed 58 cases of various disorders with CSMMs separated by $<100 \mathrm{nt}^{8}$. In the context of multiple mutations, a few cases of UCMD with compound heterozygous mutations were reported ${ }^{9,10}$.

In our case, both mutations were located at splice acceptor sites. The first mutation, c.6283-1G $>$ T, was located at the splice acceptor site of intron 17 and was predicted to cause the skipping of exon 18. The second mutation, c.6310-2 A > T, was located at the splice acceptor site of intron 18. A mutation located at the splice site towards the $\mathrm{N}$-terminal end of the triple helical domain was previously reported to cause single exon skipping ${ }^{10}$. Therefore, c.6310-2 $\mathrm{A}>\mathrm{T}$ was also predicted to cause the skipping of exon 19. Unexpectedly, c.6310-2 A > T caused the activation of the cryptic splice sites. In this case, the resultant mRNAs from c.6283-1 G > T and c.6310-2 A > T skipped exon 18 and preserved exon 19 while inserting the latter part of the intron 18 sequence. We assumed that this might be due to the difference in the number of ESEs and ISEs. In silico analysis revealed that ESE numbers in exon 18 were fewer than those in exon 19. In contrast, the ISE a. Normal allele

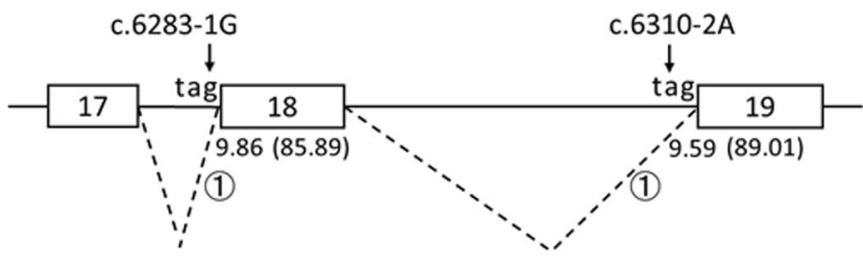

b. Mutant allele

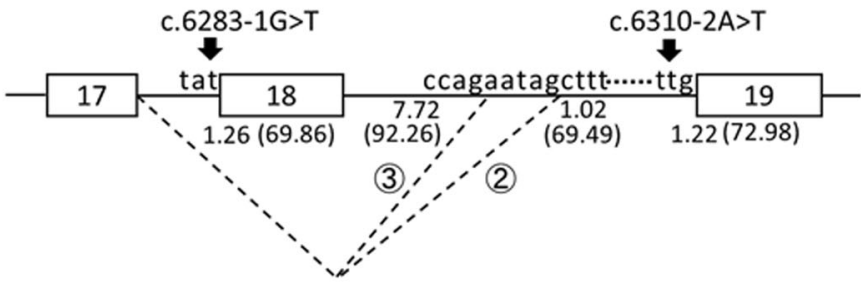

c. mRNAs

(1)

\begin{tabular}{|l|l|l|}
\hline 17 & 18 & 19 \\
\hline
\end{tabular}

(2)

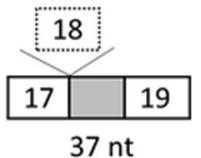

(3)

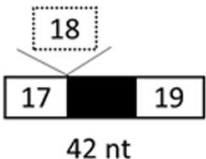

Fig. 2 Schematic representation of the splicing patterns identified in the index case. Thin and bold arrows represent mutations. a Normal COL6A3 allele in the index case. b Mutant COL6A3 allele with two closely spaced mutations. c COL6A3 mRNAs. The dotted lines indicate the splicing events. Splicing pattern (1): Normal mRNA was produced from the normal allele (a, c(1). Splicing patterns (2) and (3): The c.6283-1 G>T mutation resulted in the skipping of exon 18, whereas the c.6310-2 A > T mutation activated the cryptic splice sites in the 37- and 42-nt sequences upstream of exon 19. The MaxEntScan and Shapiro's score (in parentheses) of each splice acceptor site are depicted below each authentic and cryptic splice acceptor site. Both scores of the authentic splice acceptor sites were decreased by these mutations. Boxes and lines indicate exons and introns, respectively, and the numbers in the boxes indicate the exon number. Gray and black boxes represent the 37- and 42-nt sequences of the latter part of intron 18, respectively, and the dotted box indicates the skipped exon. Partial nucleotide sequences of the introns are shown above the line 
numbers in intron 18 were greater than those in intron 19. Thus, this analysis could not fully explain the skipping of exon 18 and the preservation of exon 19. Further research is needed to explain this phenomenon.

The results of electrophoresis and semiquantitative analysis by capillary electrophoresis revealed that the major mRNA products included the 42-nt sequence that maintained the reading frame. Since the amount of minor product was markedly small, we could not detect any bands. Thus, the findings suggest that the abnormal protein from the major product acts in a dominant fashion and that the minor product is too scarce to function.

Traditionally, UCMD was considered to be an autosomal recessive condition with severe clinical manifestations, whereas $\mathrm{BM}$ was considered to be an autosomal dominant condition with mild clinical outcomes. However, whether a condition is autosomal dominant or autosomal recessive is distinguished from the mutation patterns. Usually, cases that demonstrate heterozygous mutations are considered to be dominant conditions, whereas homozygous and compound heterozygous mutations are considered to be recessive conditions. However, in cases with two mutations, it is sometimes unclear from published reports whether these mutations are located in the same allele or in different alleles. Therefore, we suggest the existence of more undiagnosed dominant cases with multiple mutations.

\section{HGV Database}

The relevant data from this Data Report are hosted at the Human Genome Variation Database at https://doi.org/10.6084/m9.figshare.hgv.2576

\section{Acknowledgements}

We thank Ms. Yukako Yamashita for her technical assistance. This work was supported by a Grant-in-Aid for Scientific Research from the Ministry of Education, Culture, Sports, Science and Technology of Japan [grant numbers 16K21524, 2016] and Grant-in-Aid for Researchers, Hyogo College of Medicine, 2015

\section{Author details}

'Department of Pediatrics, Hyogo College of Medicine, Nishinomiya, Japan. ${ }^{2}$ Department of Pediatrics, Kobe University Graduate School of Medicine, Kobe, Japan. ${ }^{3}$ Department of Pathology and Applied Neurobiology, Graduate Schoo of Medical Science, Kyoto Prefectural University of Medicine, Kyoto, Japan. ${ }^{4}$ Department of Neuromuscular Research, National Institute of Neuroscience, National Center of Neurology and Psychiatry, Tokyo, Japan

\section{Conflict of interest}

The authors declare that they have no conflict of interest.

\section{Publisher's note}

Springer Nature remains neutral with regard to jurisdictional claims in published maps and institutional affiliations.

Received: 21 January 2019 Revised: 25 March 2019 Accepted: 2 April 2019. Published online: 26 April 2019

\section{References}

1. Baker, N. L. et al. Dominant collagen VI mutations are a common cause of Ullrich congenital muscular dystrophy. Hum. Mol. Genet. 14, 279-293 (2005).

2. Gualandi, F. et al. Autosomal recessive Bethlem myopathy. Neurology. $\mathbf{7 3}$, 1883-1891 (2009).

3. Nishikawa, A., Mitsuhashi, S., Miyata, N. \& Nishino, I. Targeted massively paralle sequencing and histological assessment of skeletal muscles for the molecular diagnosis of inherited muscle disorders. J. Med. Genet. 54, 104-110 (2017).

4. Surono, A. et al. Circular dystrophin RNAs consisting of exons that were skipped by alternative splicing. Hum. Mol. Genet. 8, 493-500 (1999).

5. Yeo, G. \& Burge, C. B. Maximum entropy modeling of short sequence motifs with applications to RNA splicing signals. J. Comput. Biol. 11, 377-394 (2004).

6. Shapiro, M. B. \& Senapathy, P. RNA splice junctions of different classes of eukaryotes: sequence statistics and functional implications in gene expression. Nucleic Acids Res. 15, 7155-7174 (1987).

7. Yeo, G. W. et al. Identification and analysis of alternative splicing events conserved in human and mouse. Proc. Natl Acad. Sci. USA 102, 2850-2855 (2005).

8. Chen, J. M., Ferec, C. \& Cooper, D. N. Closely spaced multiple mutations as potential signatures of transient hypermutability in human genes. Hum. Mutat. 30, 1435-1448 (2009)

9. Lampe, A. K. et al. Automated genomic sequence analysis of the three collagen VI genes: applications to Ullrich congenital muscular dystrophy and Bethlem myopathy. J. Med. Genet. 42, 108-120 (2005).

10. Lampe, A. K. et al. Exon skipping mutations in collagen VI are common and are predictive for severity and inheritance. Hum. Mutat. 29, 809-822 (2008). 